\title{
Lymphadenopathy in a tuberculosis-endemic area: Diagnostic pitfalls and suggested approach
}

\author{
K Antel, MB ChB, MMed (Int Med), FCP (SA), Cert Clin Haematology (SA); E Verburgh, MD, PhD (KUL) \\ Division of Haematology, Department of Medicine, Faculty of Health Sciences, University of Cape Town, South Africa
}

Corresponding author: K Antel (katherineantel@gmail.com)

Lymphadenopathy is a common presenting complaint, or it may be an incidental finding during examination of a patient. This review focuses on the diagnostic difficulty, common misconceptions and tests that can be applied in the investigation of lymphadenopathy. A diagnostic approach is suggested with regard to the most important causes of peripheral, persistent $(>3$ weeks) and significant $(>1 \mathrm{~cm})$ lymphadenopathy, i.e. tuberculosis, lymphoma and disseminated malignancy.

S Afr Med J 2019;109(10):712-714. https://doi.org/10.7196/SAMJ.2019.v109i10.14361

Tuberculosis (TB) is the most common cause of asymptomatic cervical lymphadenopathy in South Africa (SA). It is also a 'great masquerader', as it features nonspecific symptoms that can be confused with a number of other diseases. In a TB-endemic area, where the disease is front-of-mind for clinicians, lymphadenopathy may be misattributed to TB and obscure a cancer diagnosis or other cause. In particular, the misdiagnosis of lymphoma as TB has been highlighted in a number of African studies, which show that $25-85 \%$ of lymphoma patients are on TB therapy at the time of lymphoma diagnosis, the majority of whom did not have proven TB. ${ }^{[1-4]}$ Further complicating diagnosis, people living with HIV (PLHIV) are at an increased risk of both TB and lymphoma (as well as other malignancies such as Kaposi sarcoma, which may involve the lymph nodes). The increased risk of lymphoma persists despite antiretroviral treatment (ART), ${ }^{[5]}$ and in highly developed countries, lymphoma is the leading cause of HIV mortality. ${ }^{[6,7]}$

Challenges in the diagnosis of lymphoma have been highlighted and lymphoma has been identified as having the second longest time from symptom onset to diagnosis of any malignancy. ${ }^{[8]}$ Numerous barriers to the diagnosis of lymphoma are described, such as the insidious onset of symptoms and lack of specificity for diagnosis; lack of a distinct referral pathway; low sensitivity of the fine-needle aspiration (FNA) for lymphoma; and barriers in obtaining a lymph node biopsy. ${ }^{[9,10]}$ In TB-endemic areas, there are further barriers to $\mathrm{TB}$ misdiagnosis, which occur owing to overlapping symptoms (cough, loss of weight and night sweats); investigation findings (cytopenias, pleural effusions and hypodense lesions in the spleen); and nonspecific cytological findings (poorly formed granulomas that can be seen in both diseases). Furthermore, both TB and lymphoma have been described as having an onset diagnosed after immune reconstitution (TB/lymphoma immune reconstitution inflammatory syndrome (IRIS)), which may overlap in clinical presentation. Lastly, an important relationship between these diseases is that lymphoma is associated with a high risk of TB, possibly due to immune suppression, with a hazard ratio of $2.7(p<0.01)$ for developing TB compared with population controls. ${ }^{[11]}$ The relationship between HIV, TB and lymphoma places PLHIV in a TB-endemic area at an especially vulnerable risk of misdiagnosis of lymphoma (or other malignancy) as $\mathrm{TB}$.
The misdiagnosis of lymphoma or any other malignancy as TB adenitis results in delayed diagnosis and poorer outcome in lymphoma patients with more advanced disease at presentation. ${ }^{[12]}$ Making a timely and accurate diagnosis of the underlying cause for adenopathy is therefore critical, especially considering that lymphoma typically involves younger patients and is highly curable in the early stages.

\section{Commonly performed tests on lymph node tissue, applications and limitations}

Smear for acid-fast bacilli in tuberculosis

An air-dried smear for acid-fast bacilli (AFB) is a commonly performed investigation for the diagnosis of TB adenitis, as it is easy to perform from an FNA and has a quick turn-around-time (TAT). However, the test is limited by a poor sensitivity of $8-35 \%{ }^{[13-16]}$ When positive, the test is useful, as it is highly specific, with the AFB identified signifying Mycobacterium tuberculosis (adenitis due to other types of AFB are very uncommon and typically only seen in non-bacille Calmette-Guérin (BCG)-immunised children). ${ }^{[17]}$ Similar to sputum, AFBs on lymph node aspirate have been out-performed by the newer nucleic acid amplification tests for TB, such as the GeneXpert MTB/RIF (Cepheid, USA) (discussed below) and should be superseded by these testing methods.

\section{Fine-needle aspiration for cytology}

Fine-needle aspiration for cytology (FNAC) is a poor test for the diagnosis of lymphoma, especially in the absence of flow cytometry and immunohistochemistry. At our institution, we have shown that cytological findings are 'suggestive of lymphoma' in only $11 \%$ of lymphoma cases ${ }^{[12]}$ Cytology also has a long TAT, is labour intensive and is expensive. Despite these limitations, misconceptions around its use abound, as demonstrated by a cohort from our institution showing that an FNAC is often repeated when the clinician suspects lymphoma and the first test was negative. ${ }^{[12]}$ There are numerous articles on the use of FNAC in the diagnosis of lymphoma, and wide variations in reported accuracy. A recent meta-analysis reported a 'median actionable diagnosis' of $74 \%$ for FNAC, ${ }^{[18]}$ but it is important to note that the meta-analysis did not attempt to address diagnostic accuracy. The single article in this meta-analysis that discussed 
diagnostic accuracy by centralised review of specimens submitted for a clinical trial (i.e. FNAC and excisional biopsy results independently correlated) and that reflected community practice (i.e. FNACs performed by different pathologists at different centres), reported only $12 \%$ diagnostic accuracy of FNAC for lymphoma, and $29 \%$ when immunophenotyping was included. ${ }^{[19]}$

Cytology has a higher sensitivity for metastatic malignancy involving a lymph node, but there are also highly variable sensitivity rates reported in the literature. Even in a perioperative setting of patients undergoing surgery for breast cancer, the false-negative rate of FNA of axillary lymph nodes (under ultrasound guidance) has been shown to be too high (30\%) to replace excision biopsy. ${ }^{[20]}$ Due to the low negative predictive value of the FNAC from a lymph node, especially in lymphoma, the test may be useful where disseminated malignancy is highly suspected, but it should not be employed as a 'rule-out' test for either lymphoma or malignancy.

\section{GeneXpert MTB/RIF for tuberculosis on a lymph node}

A GeneXpert MTB/RIF assay on lymph node tissue was recently recommended by the World Health Organization (WHO) as the first-line investigation in suspected ТB lymphadenitis. This follows a Cochrane review showing a pooled sensitivity and specificity against culture of $87 \%$ (range 36 - 100\%) and 86\% (range 39 - 100\%), respectively. ${ }^{[2]}$ GeneXpert MTB/RIF has recently been superseded by the GeneXpert MTB/RIF Ultra assay (Ultra), which has greater sensitivity for the detection of $M$. tuberculosis complex owing to a lower limit of detection (Ultra $15.6 \mathrm{CFU} / \mathrm{mL}$ v. Xpert $114 \mathrm{CFU} / \mathrm{mL}$ ). The greatest use of the Ultra is in paucibacillary samples, such as those from lymph node tissue. These tests are cartridge based and have a fast TAT of $24 \mathrm{~h}$. These can be performed from an FNA by flushing the needle and syringe with $1-2 \mathrm{~mL}$ normal saline (even when the syringe and needle appear empty macroscopically) and by sending it in a clean specimen container, or by sending a small amount of tissue obtained by biopsy.

\section{Culture of material obtained by fine-needle aspiration or biopsy for tuberculosis}

Culture for $M$. tuberculosis from lymph node tissue and FNA performs poorly owing to a paucibacillary environment, harsh decontamination procedures and potential sampling error ${ }^{[17,22]}$ The sensitivity of mycobacterial culture from FNA may vary depending on whether the node is caseous. It has been variably reported, but is $\sim 17 \%$ with culture of an FNA, following excisional biopsy at 40 $60 \%{ }^{[17]}$ Other limitations of culture in practice are a very long TAT of up to 6 weeks, making the test impractical as an initial diagnostic procedure. The usefulness of culture on lymph node is therefore on tissue (rather than FNA).

\section{Core biopsy for diagnosis of lymphoma}

We refer to core biopsy with a wide needle, i.e $14 \mathrm{G}$ or $16 \mathrm{G}$, which is different from the fine-needle aspiration biopsy (FNAB) commonly referred to in clinical practice. Core biopsy of lymph nodes is a minimally invasive technique that can obviate the need for excision biopsy. Core biopsy is useful for diagnosing the aetiology of lymphadenopathy in HIV-infected patients. ${ }^{[23]}$ It is more frequently employed as the first step in tissue sampling in patients with suspected lymphoma, as it is safe and has up to an $89 \%$ diagnostic yield with a $14 \mathrm{G}$ or $16 \mathrm{G}$ needle. An automated device ('gun biopsy') has been safely and successfully used as a method of performing a core biopsy in patients with lymphoma, ${ }^{[24]}$ as well as in HIV and TB lymphadenitis patients. ${ }^{[23]}$ The automated device is preferred because the tissue cylinder of the biopsy retains the architecture of the tissue, and the speed of the cutting mechanism provides a clean edge with less shearing artefact. ${ }^{[24]}$ The procedure can be performed in the consulting room and has a low risk of complications. In our practice, we have found it difficult to perform in cases where a lymph node is $<15 \mathrm{~mm}$; an excision biopsy is then preferable. Where possible, a core biopsy should be performed under ultrasound guidance.

\section{Excision biopsy}

An excision biopsy remains the optimal choice for histological evaluation, but has significant cost (surgical time and expertise) and anaesthetic and surgical risks, even though the risks are low. An excision biopsy allows for the entire nodal architecture to be evaluated, as well as providing ample tissue for immunohistochemical staining, which might influence the ability to subtype the lymphoma.

\section{Conclusions and suggested diagnostic approach}

Patients with lymphadenopathy are at high risk of delayed diagnosis or misdiagnosis of the underlying disease, despite improvement in diagnostic tests. Currently, new-generation TB tests have not been incorporated into widely practised diagnostic algorithms and there is a need to educate clinicians and students about these tests and techniques. Where a lymph node is amenable to biopsy, a presumptive course of TB therapy in the era of new TB diagnostic tests is an unnecessarily hazardous practice. The risks of presumptive TB therapy include not only delaying making the correct diagnosis with the resultant morbidity, but also the potential for harmful sideeffects from TB drugs and failure to diagnose drug-resistant TB.

A more challenging situation is where lymphadenopathy is in an anatomical area where it is difficult to perform a biopsy. In these instances, a bronchoscopy/mediastinoscopy or laparoscopic biopsy should be performed, if possible, and there should be early involvement of the haematologist for diagnostic assistance, as ancillary tests such as flow cytometry or bone marrow biopsy may be useful in selected cases. In the few cases where tissue cannot be obtained due to site or technical difficulties, and patients with adenopathy are administered empirical TB therapy, they require close review to ascertain unequivocal response within 2 weeks, preferably by the clinician who initiated TB treatment.

We suggest a very simple approach to peripheral lymphadenopathy that can be followed in most adults presenting with an enlarged peripheral lymph node. In most patients, start with a GeneXpert (or Ultra) with FNA and check the results in 2 days. If the FNA GeneXpert is negative, proceed to a core biopsy with a $14 \mathrm{G}$ needle for histological evaluation (preferably providing $2-4$ core biopsy specimens), repeat the GeneXpert and request TB culture on the tissue obtained. If the core biopsy is non-diagnostic, refer the patient for a surgical excision biopsy. FNAC may be reserved for patients strongly suspected of having head and neck cancer or disseminated malignancy. In patients in whom TB is an unlikely diagnosis, an FNA need not be carried out and a core biopsy with both GeneXpert and histological evaluation is a reasonable first investigation.

Declaration. This article is based on a study done by KA in partial fulfilment of her $\mathrm{PhD}$ thesis.

Acknowledgements. The haematology clinic in E5 for allowing us to implement a rapid-access lymph node biopsy clinic. Prof. Gary Maartens for his expertise and guidance with tuberculosis diagnosis and diagnostic research. 
Author contributions. KA wrote the manuscript. EV supervised the research and edited the manuscript.

Funding. KA has been supported by the US National Institutes of Health, the Fogarty International Center (grant number D43-TW010345 and D43-TW010543), and the Peter Jacobs Bursary Trust.

\section{Conflicts of interest. None.}

1. Swart L, Novitzky N, Mohamed Z, Opie J. Hodgkin lymphoma at Groote Schuur Hospital, South Africa: The effect of HIV and bone marrow infiltration. Ann Hematol 2019;98(2):381-389. https://doi. org/10.1007/s00277-018-3533-0

2. Puvaneswaran B, Shoba B. Misdiagnosis of tuberculosis in patients with lymphoma. S Afr Med J 2013;103(1):32-33. https://doi.org/10.7196/SAMJ.6093

3. Buyego P, Nakiyingi L, Ddungu $\mathrm{H}$, et al. Possible misdiagnosis of HIV associated lymphoma as tuberculosis among patients attending Uganda Cancer Institute. AIDS Res Ther 2017;14(1):13. https:// doi.org/10.7196/SAMJ.6093

4. De Witt P, Maartens DJ, Uldrick TS, Sissolak G. Treatment outcomes in AIDS-related diffuse large B-cell lymphoma in the setting roll-out of combination antiretroviral therapy in South Africa. J Acquir Immune Defic Syndr 2013;64(1):66-73. https://doi.org/10.1097/QAI.0b013e3182a03e9

5. Yarchoan R, Uldrick TS. HIV-associated cancers and related diseases. N Engl J Med 2018;378(11):1029-1041. Yarchoan R, Uldrick TS. HIV-associated ca
https://doi.org/10.1056/NEJMra1615896

6. Achenbach CJ, Cole SR, Kitahata MM, et al. Mortality after cancer diagnosis in HIV-infected individuals treated with antiretroviral therapy. AIDS 2011;13(255):691-700. https://doi.org/10.1097/ QAD.0b013e3283437f77

7. Simard EP, Engels EA. Cancer as a cause of death among people with AIDS in the United States. Clin Infect Dis 2010;51(8):957-962. https://doi.org/10.1086/656416

8. Howell DA, Smith AG, Roman E. Lymphoma: Variations in time to diagnosis and treatment. Eur I Cancer Care (Engl) 2006;15(3):272-278. https://doi.org/10.1111/j.1365-2354.2006.00651.x

9. Howell DA, Smith AG, Roman E. Referral pathways and diagnosis: UK government actions fail to recognize complexity of lymphoma. Eur J Cancer Care (Engl) 2007:16:529-532. https://doi.org/10.1111/ j.1365-2354.2007.00789.x

10. Nikonova A, Guirguis HR, Buckstein R, Matthew C. Predictors of delay in diagnosis and treatment in diffuse large B-cell lymphoma and impact on survival. Br J Haematol 2015;168:492-500. https://doi. org/10.1111/bih. 13150

11. Ganzel C, Silverman B, Chemtob D, Ben Shoham A, Wiener-Well Y. The risk of tuberculosis in cancer patients is greatest in lymphoma and myelodysplastic syndrome/myeloproliferative neoplasm: A larg population-based cohort study. Leuk Lymphoma 2019;60(3):720-725. https://doi.org/10.1080/1042819 4.2018.1499904
12. Antel $\mathrm{K}$, Levetan $\mathrm{C}$, Mohamed $\mathrm{Z}$, et al. The determinants and impact of diagnostic delay in lymphoma in a TB and HIV endemic setting. BMC Cancer 2019;19(1):384. https://doi.org/10.1186/s12885-019in a TB

13. Bem C, Patil PS, Elliott AM, Namaambo KM, Bharucha H, Porter JD. The value of wide-needle aspiration in the diagnosis of tuberculous lymphadenitis in Africa. AIDS 1993;7(9):1221-1225.

14. Ellison E, Lapuerta P, Martin SE. Fine needle aspiration diagnosis of mycobacterial lymphadenitis. Sensitivity and predictive value in the United States. Acta Cytol 1999;43(2):153-157. https://doi org $/ 10.1159 / 000330969$

15. Fain O, Lortholary O, Djouab M, et al. Lymph node tuberculosis in the suburbs of Paris: 59 cases in adults not infected by the human immunodeficiency virus. Int J Tuberc Lung Dis 1999:3(2):162-165.

6. Asimacopoulos EP, Berry M, Garfield B, et al. The diagnostic efficacy of fine-needle aspiration using Asimacopoulos EP, Berry M, Garfield B, et al. The diagnostic efficacy of fine-needle aspira
cytology and culture in tuberculous lymphadenitis. Int J Tuberc Lung Dis 2010;14(1):93-98

17. Fontanilla JM, Barnes A, von Reyn CF. Current diagnosis and management of peripheral tuberculous lymphadenitis. Clin Infect Dis 2011;53(6):555-562. https://doi.org/10.1093/cid/cir454

18. Frederiksen JK, Sharma M, Casulo C, Burack WR. Systematic review of the effectiveness of fineneedle aspiration and/or core needle biopsy for subclassifying lymphoma. Arch Pathol Lab Med 2015;139(2):245-251. https://doi.org/10.5858/arpa.2013-0674-RA

19. Hehn ST, Grogan TM, Miller TP. Utility of fine-needle aspiration as a diagnostic technique in lymphoma. J Clin Oncol 2004;22(15):3046-3052. https://doi.org/10.1200/JCO.2004.02.104

20. Kane G, Fleming C, Heneghan $\mathrm{H}$, et al. False-negative rate of ultrasound-guided fine-needle aspiration cytology for identifying axillary lymph node metastasis in breast cancer patients. Breast J 2019;13(001):1-5. https://doi.org/10.1111/tbj.13402

21. Kohli M, Schiller I, Dendukuri N, et al. Xpert" MTB/RIF assay for extrapulmonary tuberculosis an rifampicin resistance. Cochrane Database Syst Rev 2018;(8):CD012768. https://doi.org/10.1002/14651858. CD012768.pub2

22. Wright CA, Hesseling AC, Bamford C, Burgess SM, Warren R, Marais BJ. Fine-needle aspiration biopsy: A first-line diagnostic procedure in paediatric tuberculosis suspects with peripheral lymphadenopathy? Int J Tuberc Lung Dis 2009;13(11):1373-1379.

23. Wilson D, Nachega JB, Chaisson RE, Maartens G. Diagnostic yield of peripheral lymph node needlecore biopsies in HIV-infected adults with suspected smear-negative tuberculosis. Int J Tuberc Lung Dis 2005;9(2):220-222

24. Demharter J, Miller P, Wagner T, Schlimok G, Haude K, Bohndorf K. Percutaneous core-needle biopsy of enlarged lymph nodes in the diagnosis and subclassification of malignant lymphomas. Eur Radio 2001;11(2):276-283. https://doi.org/10.1007/s003300000540

Accepted 26 August 2019. 